\title{
Pharmacokinetics of amphotericin B and flucytosine
}

\author{
AnNemarie Polak \\ Dr sc. nat.
}

\author{
Pharmaceutical Research Division, F. Hoffmann-La Roche \& Co. Ltd, Basle, Switzerland
}

\begin{abstract}
Summary
The absorption of flucytosine from the digestive tract is very good but minimal with amphotericin B. For this reason, amphotericin $B$ has to be injected intravenously. Fungistatic levels are achieved rapidly with flucytosine and slowly with amphotericin B, since the dose has to be increased slowly. Distribution of flucytosine in other body fluids is high, whereas with amphotericin $B$ it is poor. Flucytosine is excreted mainly via the kidney without metabolism, whereas amphotericin $B$ is eliminated mainly by metabolism. Therefore, amphotericin $B$ dosage does not have to be adapted to kidney function, which is the case for flucytosine. The half-life of flucytosine is short (hours), that of amphotericin B is long (days); flucytosine is haemodialysable, whereas amphotericin B, probably owing to the high protein binding, is not.
\end{abstract}

\section{Introduction}

The pharmacokinetic properties of the intravenous antifungal amphotericin $B$ and of the oral antifungal flucytosine differ fundamentally. In this paper they are first discussed individually and then their effect when used clinically alone or in combination is compared.

\section{Amphotericin B}

Amphotericin B has been used as a chemotherapeutic agent for more than 20 years. Yet investigations providing a rational pharmacokinetic basis for intravenous or intrathecal treatment are scarce and several important phenomena poorly understood. During intravenous therapy, $90 \%$ of amphotericin B disappears from the blood although only consistently low levels are found in body fluids other than serum. Moreover, the amphotericin B portion remaining in the blood is strongly bound to serum proteins, especially lipoproteins.

Dose recommendations for the safe and effective use of amphotericin B are essentially based on the tolerance of patients receiving the drug. The highest

Correspondence: Dr Annemarie Polak, c/o F. HoffmannLa Roche \& Co. Ltd, 124, Grenzacherstrasse, 4002, Basle, Switzerland. tolerated dose $(1 \cdot 0-1 \cdot 5 \mathrm{mg} / \mathrm{kg}$ body weight or $50-100$ mg) was administered daily by the early investigators. But it gradually became apparent that a double dose on every other day was more effective and better tolerated. Bindschadler and Bennett (1969) compared the amphotericin B levels in serum after a single and a double dose. At doses of from 5 to $50 \mathrm{mg}$, a correlation was found between blood levels and daily doses. As the doses were increased, inactivation or storage mechanisms seemed to disturb this correlation and limited the peak serum concentrations of the drug. As might have been expected, infusions of up to $90 \mathrm{mg}$ on alternate days gave higher peak levels than those obtained with a daily dose of $45 \mathrm{mg}$. However, the minimal concentrations were not significantly different so that a similar antifungal activity could be expected. The drug given on every other day is much better tolerated, allowing the patient a day of rest between doses. More recently, the tendency has been for comparatively low doses to be given, e.g. $0.4 \mathrm{mg} / \mathrm{kg}$ body weight daily or on alternate days.

In order to avoid the severe side effects such as pyrexia and chills, treatment with amphotericin B is usually started in small doses which are gradually increased during a period of 3-4 days. The disadvantage of this is that the production of therapeutically active serum levels is correspondingly delayed. Using a pharmacokinetic model, Atkinson and Bennett (1978) compared amphotericin B levels resulting from 2 different regimens: with the first, giving $1 \mathrm{mg}$ on the first day and 5,10 and $50 \mathrm{mg}$ on the following 3 days respectively, the serum levels necessary for antifungal activity $(0.2-0.5 \mathrm{mg} / \mathrm{l})$ were reached only on day 4 and were maintained for only $10 \mathrm{hr}$. With the second regimen $(25 \mathrm{mg}$ on days 1 and 2 , and 30 mg on day 3 ) the critical serum level was reached on day 2 , and it was possible to maintain it. The second of these regimens was therefore recommended for seriously ill patients.

Atkinson and Bennett (1978) studied carefully the pharmacokinetics of amphotericin B at the conclusion of treatment lasting 2-4 months. The distribution kinetic of amphotericin B was adequately described by a 3-compartment system: the drug is 
introduced into a central (intravascular) compartment and distributed into 2 peripheral (extravascular) compartments, of which the one is fast and the other slow to equilibrate. About $66 \%$ of the drug is located in the latter compartment when the steady state is reached. Initially, amphotericin B is rapidly eliminated, the half-life being about $24 \mathrm{hr}$. The second terminal elimination phase, however, has a half-life of 15 days, indicating the high storage potency in the slow equilibrating compartment, which has a volume of 220 litres compared with 30 litres of the other 2 compartments. The relative distribution volume is high (4 litres/ $\mathrm{kg}$ ), probably owing to the strong protein- and membrane-binding activity of amphotericin B. The amphotericin B levels of 4 patients studied by Fields, Bates and Abernathy (1970) accord well with results studied in the model by Atkinson and Bennett (1978). The only speculation is about the exact location of the 3 compartments.

Elimination of the amphotericin B methyl ester (given i.v.) was explained in a similar way to that of amphotericin B by a 3-compartment model; however, the overall elimination from serum was found to be 4 times faster than for amphotericin B. With $5 \mathrm{mg} / \mathrm{kg}$ of amphotericin B methyl ester, peak serum levels were 7-12 times higher than with $1 \mathrm{mg} / \mathrm{kg}$ of amphotericin B. In spite of this, nephrotoxicity was either absent or very weak (Jagdis et al., 1977).

Renal excretion of amphotericin B is negligible. Only $3 \%$ of the dose can be found in the urine. Pre-existing renal dysfunction had no effect on the plasma levels of the drug. Even in anuric and nephrectomized patients, the amphotericin B serum levels and its elimination are exactly the same as in normal patients. Apparently, amphotericin B is eliminated mainly by metabolic conversion or by the bile (Craven, Ribble and Drutz, 1977). However, the metabolites are not yet known.

Concentrations in other body fluids after intravenous injection of amphotericin B are rather low compared with the serum level (Table 1). On average, the level of amphotericin B reaches $0.05 \mathrm{mg} / \mathrm{l}(2-4 \%$ of the one in serum) in the spinal fluid. In the peritoneal fluid, pleura or joint, less than $50 \%$ of the serum levels are achieved. In bronchial secretion, a level of $0.16 \mathrm{mg} / \mathrm{l}$ was measured only immediately after the infusion and this declined rapidly. The concentrations in the urine are low. In the aqueous humour of one patient, a high concentration (comparable to that in serum) was measured, but considerably lower concentrations were found in rabbits with experimental uveitis. Amphotericin B also crosses the placenta (Bennett, 1975) (Table 1).

Since the concentrations of amphotericin B in spinal fluid after intravenous injection are so low, patients with meningitis are often treated with an intrathecal injection of the drug. The pharmacokinetics of intrathecally administered amphotericin B can be demonstrated using a 2-compartment model (Atkinson and Bindschadler, 1969). Amphotericin B rapidly disappears from spinal fluid after initial administration. However, after a prolonged treatment, the half-life is increased because the second compartment serves as a reservoir, and amphotericin $B$ is accumulated there. As in the serum model, the identity of this compartment or pool is unknown.

TABLE 1. Concentrations of amphotericin B after intravenous injection

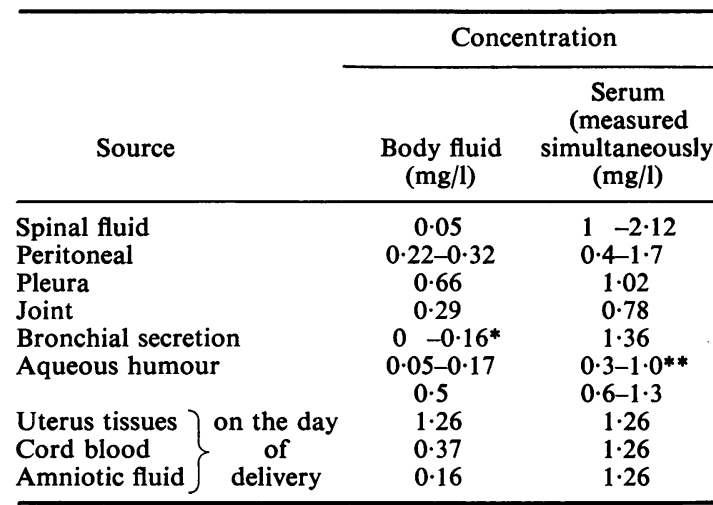

* Only immediately after injection.

** In rabbits with uveitis.

These careful studies of the pharmacokinetics of amphotericin $B$ will give a basis and guide for a more effective and better tolerated treatment of systemic mycosis with amphotericin $B$ in the future.

There are, however, still a number of unknown pharmacological properties of the drug which should be studied further, e.g. the metabolism of amphotericin B and the identity of compartments where amphotericin B is accumulated in the body.

\section{Flucytosine}

The absorption from the digestive tract is rapid and almost total. Peak levels of flucytosine were measured 90-120 min after oral administration if kidney function was normal and 4-5 hr after if it was insufficient. The delay is because of the slow elimination.

In volunteers and in patients, maximal serum concentrations in $\mathrm{mg} / \mathrm{l}$ roughly correspond to the single dose of the drug in $\mathrm{mg} / \mathrm{kg}$ body weight (e.g. 30-40 $\mathrm{mg} / \mathrm{l}$ after a single dose of $30 \mathrm{mg} / \mathrm{kg}$ [Wade and Sudlow, 1972; Schönebeck et al., 1973; Dawborn, Page and Schiavone, 1973]). For chemotherapy it is necessary to maintain a steady state concentration of between 25 and $100 \mathrm{mg} / \mathrm{l}$. Higher concentrations over a longer period are toxic (Kauffman and Frame, 1977). At lower concentrations, there is an 
increased risk of secondary resistance (Normark and Schönebeck, 1973). With normal renal function, these conditions are achieved with a dose of $50 \mathrm{mg} / \mathrm{kg}$ given every $6 \mathrm{hr}$.

Concentrations of flucytosine well above the fungistatic level are also obtained in most body fluids. In spinal fluids, $38 \pm 7 \mathrm{mg} / 1$ (71-85\% of the serum level) were estimated in more than 50 patients. In bronchial secretion of dogs and in the bile of rats, the flucytosine levels were in the same range as the serum levels. With $8-24 \mathrm{mg} / \mathrm{l}$ the levels in saliva were slightly lower than the $14-47 \mathrm{mg} / 1$ measured in serum. In the bone, $30 \%$, and in the joint fluid, $41 \%$ of the serum levels were reached. In the peritoneal fluid, $2-8 \mathrm{mg} / \mathrm{l}$ were measured simultaneously with $8-20 \mathrm{mg} / \mathrm{l}$ in the serum. In the aqueous humour, the level reached $10 \mathrm{mg} / 1$. Extremely high concentrations are achieved in the urine because most of the drug is excreted unchanged via the kidney by means of glomerular filtration as is also the case with creatinine and inulin; $97 \%$ of the dose is excreted in the urine.

The pharmacokinetic properties of flucytosine can be explained by a simple one-compartment model. The drug's half-life in patients with normal kidney function is $3-5 \mathrm{hr}$, independent of the administration route (Wade and Sudlow, 1972, 3.77 hr;
Schönebeck et al., 1973, $2.89 \mathrm{hr}$; Dawborn et al., $1973,5.44 \mathrm{hr}$ ). The relative distribution volume is between 0.6 and 0.9 litre $/ \mathrm{kg}$ in patients with normal or impaired kidney function.

However, the half-life is significantly prolonged with renal insufficiency, e.g. in a patient whose creatinine clearance was $35 \mathrm{ml} / \mathrm{min}$, the half-life was $10 \mathrm{hr}$, and it was $21 \mathrm{hr}$ in another patient with a creatinine clearance of $9 \mathrm{ml} / \mathrm{min}$. In anuric or nephrectomized patients, the average half-life was 3-4 days. Enormous accumulation occurred naturally unless the dosage was substantially reduced.

Based on a linear correlation between the elimination rate constant of flucytosine and creatinine clearance, a dosage schedule has been established for patients with renal insufficiency of any degree. The individual doses are left constant but the dose interval is extended. A creatinine clearance above $40 \mathrm{ml} / \mathrm{min}$ is still considered to be normal. With a creatinine clearance above $40 \mathrm{ml} / \mathrm{min}$, the dose is given every $12 \mathrm{hr}$. With a creatinine clearance of 20 $10 \mathrm{ml} / \mathrm{min}$, the dose is given every day. With a creatinine clearance below $10 \mathrm{ml} / \mathrm{min}$, the interval is still longer and must be adapted to the measured flucytosine levels in serum.

Dufresne (1972) studied the pharmacokinetic properties of flucytosine in spinal fluid after intra-

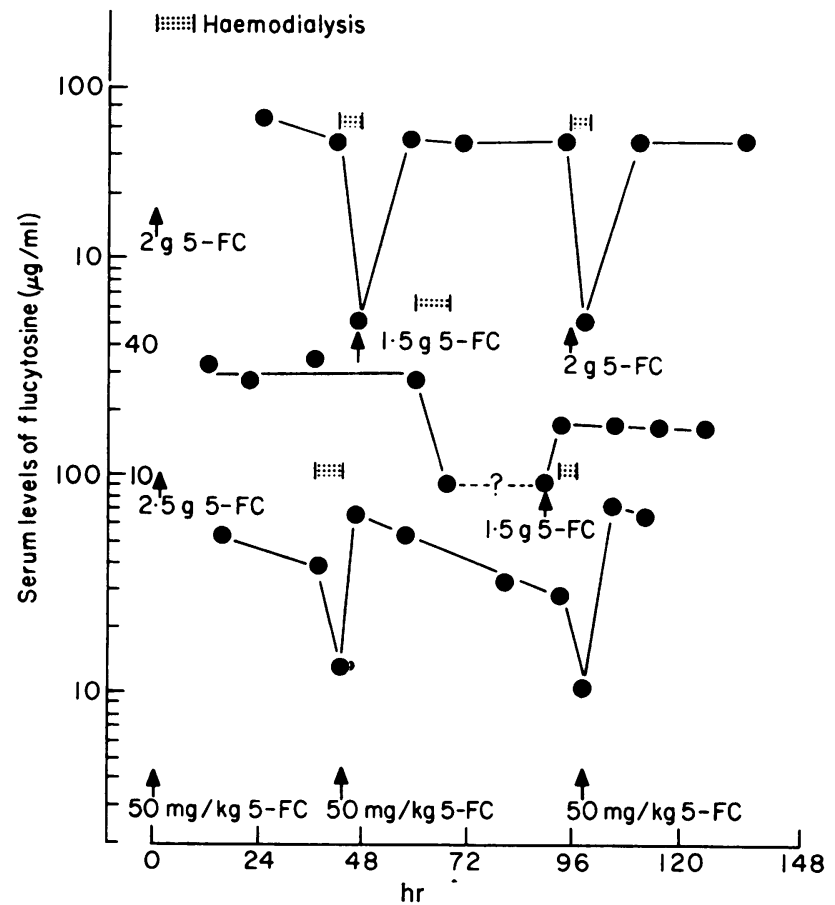

Fig. 1. Serum levels of flucytosine before and after dialysis in 3 patients. 10 Haemodialysis; 5-FC = flucytosine. 
thecal injections in one patient. After $24 \mathrm{hr}$, the concentration of flucytosine in the ventricular fluid was lower than in the lumbar fluid, independent of whether the injection was by the intraventricular or lumbar routes. The half-life was $6.6 \mathrm{hr}$ in the lumbar fluid and 7.8 in the ventricular fluid. These half-lives were significantly longer than the half-life in serum $(3.8 \mathrm{hr})$.

Flucytosine is efficiently eliminated by haemodialysis. Figure 1 shows serum levels before and after dialysis in 3 patients. Haemodialysis clearance of flucytosine was indistinguishable from that of creatinine and increased proportionally to the blood flow through the dialyser. Haemodialysis removed $66-75 \%$ of the flucytosine content of the body (Drouhet et al., 1973; Schönebeck et al., 1973; Polak, unpublished data, 1975). Peritoneal dialysis is generally less effective than haemodialysis, but there is evidence that the clearance of flucytosine by peritoneal dialysis is equal to that of creatinine by the same process.

\section{References}

Atkinson, A.J. \& Bennett, J.E. (1978) Amphotericin B pharmacokinetics in humans. Antimicrobial Agents and Chemotherapy, 13, 271.

Atkinson, A.J. \& Bindschadler, D.D. (1969) Pharmacokinetics of intrathecally administered amphotericin B. American Review of Respiratory Disease, 99, 917.

BennetT, J.E. (1975) Review of amphotericin B. In: Proceedings of the Ninth International Congress of Chemo- therapy. London, 13-19 July, 1975. Plenum Press, New York.

BindSChadler, D.D. \& BenNeTt, J.E. (1969) A pharmacologic guide to the clinical use of amphotericin B. Journal of Infectious Diseases, 120, 427.

Craven, P.C., Ribble, R.J. \& Drutz, D.J. (1977) Biliary excretion of biologically active amphotericin B. Clinical Research, 25, 374A.

Dawborn, J.K., Page, M.D. \& Schiavone, D.J. (1973) Use of 5-fluorocytosine in patients with impaired renal function. British Medical Journal, 4, 382.

Drouhet, E., Babinet, P., Chapusot, J.P. \& Kleinknecht, D. (1973) 5-fluorocytosine in the treatment of candidiasis with acute renal insufficiency. Biomedicine, 19, 408.

Dufresne, J.J. (1972) Le traitement de la méningo-encéphalite cryptococcique, intérêt de la 5-fluorocytosine. Revue Médicale de la Suisse Romande, 92, 597.

Fields, B.T., BATES, J.H. \& AbERNATHY, R.S. (1970) Amphotericin B serum concentrations during therapy. Applied Microbiology, 19, 955.

JAGDIS, F.A., HOEPRICH, P.D., LAWRENCE, R.M. \& SCHAFFner, C.P. (1977) Comparative pharmacology of amphotericin B and amphotericin B methyl ester in the non-human primate, Macaca mulatta. Antimicrobial Agents and Chemotherapy, 12, 582.

Kauffman, C.A. \& Frame, P.T. (1977) Bone marrow toxicity associated with 5-fluorocytosine therapy. Antimicrobial Agents and Chemotherapy, 11, 244.

Normark, S. \& Schönebeck, J. (1972) In vitro and in vivo 5 -fluorocytosine resistance in Candida albicans and Torulopsis glabrata. Antimicrobial Agents and Chemotherapy, 2, 114.

Schönebeck, J., Polak, A., Fernex, M. \& Scholer, H.J $\stackrel{\oplus}{+}$ (1973) Pharmacokinetic studies on the oral antimycotie agent 5-fluorocytosine in individuals with normal ando impaired kidney function. Chemotherapy, 18, 321.

WADE, D.N. \& Sudlow, G. (1972) The kinetics of 5-fluorocytosine elimination in man. Australian and New Zealand Journal of Medicine, 2, 153. 